\title{
History, Epidemiology and Clinical Characteristic of Adverse Events
}

Kozulina Irina ${ }^{1,2^{*}}$, Danilov Alexey ${ }^{2}$ and Andrianov Vladimir ${ }^{2}$

${ }^{1}$ Chair of Organization of Scientific Studies and Pharmacovigilance System, Russia

${ }^{2}$ Institute of Interdisciplinary Medicine, Moscow, Russia

${ }^{*}$ Corresponding author: Kozulina Irina, IInstitute of Interdisciplinary Medicine, Moscow, Russia, Tel: +7 (495) 7965158; E-mail: kozulina@idm.institute

Received date: July 20, 2018; Accepted date: August 23, 2018; Published date: August 30, 2018

Copyright: $\odot 2018$ Irina K, et al. This is an open-access article distributed under the terms of the Creative Commons Attribution License, which permits unrestricted use, distribution, and reproduction in any medium, provided the original author and source are credited.

\begin{abstract}
Rapid medicine and pharmacy progress bring new high-technology medical product to controlling and treating different diseases. Unfortunately, it does not correct a problem of drugs adverse events (ADE) from mild side effects till severe hyper sensitivity reactions, which can lead a new diseases and forming a temporary or total disability and death in some cases. There are about 17 thousand of different medicines in the world now, nearly $90 \%$ of them where done in last ten years. According to update data in last ten years mortality rate due to ADE takes 4-6 places in the world. In various ways the rate of hospitalization due to $A D E$ is from $2 \%, 4 \%$ to $12 \%$. As well as, ADE increases the volume of direct and indirect cost of patient treatment. In USA treatment of patients with ADE is about 30, 1 milliard dollars per year. According to Sultana, treatment cost for one person with one ADE averages about $2262 \$$. According to Wester et al. most often ADE are gastrointestinal bleeding, cerebrospinal bleeding, cardiovascular accidents, and other bleeding and renal disorders. Primary ADE rate among medicines belong to nonsteroidal antiinflammatory drug. According to meta-analysis prospective studies of Lazarou et al. as a result of ADE there were more than 100000 death cases. Makary determined that most commonly ADE was because of medical error.
\end{abstract}

Keywords: Adverse drug events; Mortality; Chloroform; Thalidomide; Epidemiology

\section{History}

The first ADE report was published in 1898 in Lancet about chloroform anesthesia. At the beginning of $20^{\text {th }}$ century in USA after sulphanilamide liquid on ethylene glycol base therapy there was about 100 children death cases, wherein it was known about life threatening characteristic of the substance. In 1938 after the tragedy the demand of safety evidence of new drug before output it in the market was added to Federal Law «Food, Drug and Cosmetic Act» (FDA), USA [1-9].

One of the major dire consequences was Thalidomide tragedy. In 1954 German pharmaceutical company Chemie Grünenthal conducted the research of budget method of antibiotics production through peptides. During this study Thalidomide was produced. The scientist thought that the new drug was safety because overdose kept animals alive. In 1955 drug company began to give the drugs free to HCPs and patients indicate good calm and soporific effect. In 1957 the company began to sell the drug like Contergan in FRG, then in Great Britain in 1958. Thalidomide was used in bronchial asthma treatment, for blood pressure decreased, for migraine therapy, on sale level it was like aspirin. Thalidomide was sold in 46 European countries, in Northland, Asia, Africa and South America. However, there were no external in depended studies. In 1958 there was a letter with information, that Thalidomide was the best treatment for pregnant and nursing mothers. It became a part of advertising company in Great Britain without any studies of side drug effects on unborn child. Thalidomide became very popular for insomnia, worry, nausea therapy during pregnancy. It was the most sold out sedative drug in FRG. Since 1956 the reports about different types of periphery neuritis and other adverse events had been received to Grünenthal. The drug began to sell out by prescription only. In December 1956 in Grünenthal employee family was born a daughter without ears. He gave his pregnant wife Thalidomide before it's official sale was start. But nobody observed this malformation with this drug. Nevertheless, from the date of official used of Thalidomide in pregnant women number of children with malformations was increased seriously. In 1961 German HCP Hans-Rudolf Wiedemann said about epidemiology. A little bit later professor W. Lenz in Germany and doctor McBrite in Australia noticed this with Thalidomide mother's therapy in the early stages of pregnancy. As a result of Thalidomide therapy there were about 40000 patients with periphery neuritis and about 8000-12000 newborn with different kind of malformation, nearly 5000 alive and became invalid $[10,11]$.

\section{Regulatory Authorities}

This dire consequences of this tragedy necessitated a major practice reviewer and requirement strengthening to drug licensing. Event dire in USA lead to amendment of drug legislation in 1962 whereby was induced demand of realization of pre-licensure trial for the purpose of safety and efficacy of new drugs [12]. Between 1965-1970 there were a lot of new meetings and resolutions, in 1967 WAO produced an international program of drug monitoring and then pharmacovigilance system for drug safety and efficacy monitoring [1]. In 1968 in Uppsala (Sweden) was opened WAO center of international drug monitoring (UMC) [12]. Pharmacovigilance (PV) is a system which including scientific studies and other types of activity associated with assessment, understanding and prevention of adverse drug events or other problems with drugs [13].

\section{Primary PV activities}

- To determine potential risks associated with medicines and evaluate risk-benefit ratio. 
- To sell the line of safety, effectiveness and rational use of medicines.

- To form more deliberate policy of medicines using among HCPs and patients.

Advance of this goal is possible only by team work of HCPs, media, patients and head of pharm industry. Since 1969 there was demanded department of regulation of adverse events reports and then in 1997 there were opened 20 first region centers of RZN (Federal Service for Surveillance in Healthcare (Roszdravnadzor), including Federal center of drugs side effects studying of Ministry of Health of the Russian Federation. At that year Russia was included in WHO program for international drug safety monitoring in 2007 Federal center of drug safety monitoring was opened due to RZN protection [14-16].

\section{Adverse Events Classification}

According to GCP, ADE is any untoward medical occurrence in a patient or clinical investigation subject administered a pharmaceutical product and which does not necessarily have a causal relationship with this treatment. An ADE can therefore be any unfavourable and unintended sign (including an abnormal laboratory finding), symptoms, or diseases temporally associated with the use of a medicine (investigational) product, whether or not related to the medicine (investigational) product (see the ICH Guidance for Clinical Safety Data Management: Definitions and Standards for Expedited Reporting). All ADE according that mechanism, clinical features and prognoses by WHO recommendation are divided into:

- Dose-depended and expected (can divided any patient on medication).

- Dose-independed and unexpected (for sensitive patients).

- Effects of long-term treatment (less effectiveness, resistance).

- Long term effect $[17,18]$.

It is necessary to evaluate cause-effect relationship between medicine administration and ADE.

\section{Epidemiology and ADE Clinical Characteristics}

Statistical investigations most common causes of hospitalization and patient's death by drug adverse reactions, spectrum of the most dangerous medicines are regularly ongoing in different countries. Wester et al. was analyzed 49 death cases in three Sweden regions since January 1 till December 31 in 2001 by drugs AE. Most commonly the death was because of gastrointestinal bleeding (37\%), central cerebrospinal bleeding (29\%) and other bleedings (abdominal, respiratory and other) (4\%), nearly $2 \%$ because of cardiovascular accidents (bradycardia, cardiomyopathy, hypotension, pulmonary embolism, cardiac failure), about $2 \%$ because of nephrosis disorder. Most of ADE progressed after antithrombotic drugs therapy like warfarin, heparin, enoxaparin and etc. (33\%), blood platelet aggregation inhibitors (aspirin, dipyridamole, clopidogrel) $-43 \%$ cases, non-steroidal anti-inflammatory drugs (diclofenac, ketoprofen, celecoxib) $-18 \%$, antidepressant medication $14 \%$, analgesics $2 \%$, systemic antibiotics $2 \%$ [4].

Totally there were analyzed 4331 cases of hospitalization, duration not longer than 8 days the patients simultaneously took on 4-9 medications. As a result, $48 \%$ hospitalization were because of ADE, in $11 \%$ of cases the reaction was clinically confirmed. Most of ADE were after chemotherapy (30, 7 cases), antibiotics (2, 8 cases), antivirals medication $(2,6$ cases $)$ and non-steroidal anti-inflammatory drugs ( 1 ,
8 cases). From 131 patients with gastrointestinal disorders, 89 had nausea and vomiting after chemotherapy, 21 had gastro-intestinal bleeding and/or tumor according with non-steroidal antiinflammatory treatment. From 112 hospitalized patients with hemopoietic system defects there were 97 cases of leukopenia/ agranulocytosis, anemia (8 cases), pancytopenia (4 cases), and thrombocytopenia (6 cases). Those reactions mostly developed after chemotherapy (84 cases), clotrimazole (7 cases), other antibiotics (6 cases) and antiviral (6 cases). There were 93 patients with skin reactions, 79 of them had itching and rash after antibiotic therapy (47 cases), non-steroidal anti-inflammatory drugs (6 cases), diuretics (4 cases). 11 patients had maculo-papular rash, hypersensitivity reaction, immune thrombocytopenic purpura and big hematoma. About 27 hospitalizations were because of bradycardia, atrial fibrillation, and arrhythmia, hypotension after B-adrenergic antagonists, antiarrhythmics, antidepressant and diuretics. The reason of 25 hospitalizations was massive hematoma after anticoagulants, 11 cases of osteoporosis after steroids.

Nephrosis disorders like acute renal failure most of all was after non-steroidal anti-inflammatory drugs, neurology disorders like dyskinesia, tremor and peripheric neuropathy after neuroleptics. In Switzerland Fattinger et al. [19] were estimate the structure, critical parameter and treatment of ADE with the help of electronic data base of two large medical centers. Highest death cases were among the patients with chemotherapy because of induced agranulocytosis and infection [19]. In 2015 Bouvy was published summary results of prospective and post-registration observation studies since January 1, 2003 till September 3, 2014 for assessment of ADE epidemiology in Europe. As a result, nearly $5 \%$ of hospitalization was because of ADE reactions, nearly $10,1 \%$ of patients had ADE during hospitalization. In $72 \%$ of all studies there was information about fatal adverse reaction, no information about children death rate, may be because of low risk rate of children death after $\mathrm{AE}$ [20].

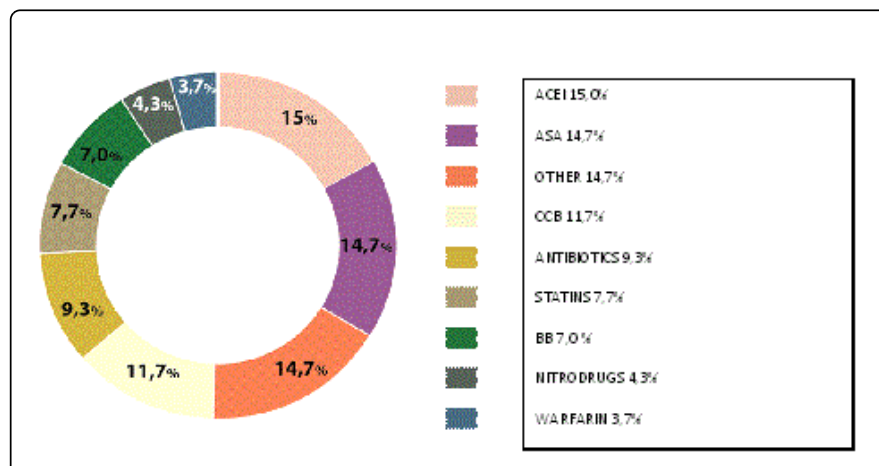

Figure 1: Frequency reactions array according to drug groups.

According to other sources from 2, $1 \%$ to $5,2 \%$ of children needed a hospitalization and more than $39 \%$ of cases were life-threatening or fatal [6]. So, nearly 83,1 million people per year is hospitalizing in 31 European countries with total population 504 million people. The rate of death cases after ADE during hospitalization after therapy is about $0,5 \%$, it is nearly 419000 people every year. In USA this rate is nearly 197000 people in the year [20].

In 2016 the State Scientific-Research Center for Preventive Medicine, Moscow, presented the first results of drugs ADE research according to data of outpatient register of primary patients included to the register since January, 2011 till August, 2011. About 223 of the 
patients had experienced different kind of ADE. Most of this patient had arterial hypertension, chronic heart failure and ischemic heart disease. Most ADE were after angiotensin-converting-enzyme inhibitor therapy, acetylsalicylic acid on the average about $15 \%$ from total number of cases, $11,7 \%$ was after calcium channel blocker therapy, about 9, 3\% after antibiotics, 7, 7\% after statins (Figure 1 and 2).

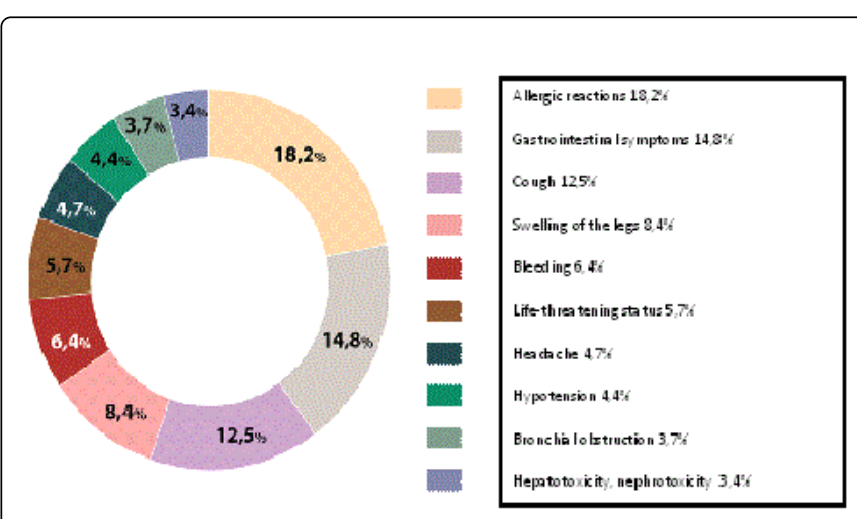

Figure 2: Adverse events partitions according to clinical response.

\section{Conclusion}

Based on this data, we can confirm that most often ADE is symptoms of gastrointestinal tract disorder, cardiovascular system disorder, skin reactions. The most aggressive drugs are non-steroidal anti-inflammatory drugs, drugs for cardiovascular disorder treatment and chemotherapy. A special group of risk consists of patients who simultaneous take on several different groups of drugs. It is very important be careful during the process of medicines prescription and try to escape the polypharmacy. Analysis of the patient's medical history and family predisposition, adherence to the manufacturer's recommendations, understanding of pharmacodynamics and pharmacokinetics specific of different drug groups, personalized approach significantly reduces the risk of ADE developing. All information about different reaction during drug therapy should be controlling and send to regulatory authorities and pharmaceutical company timely. Pharmacovigilance system significantly affecting to the regulation process of the medicines turnover, based on safety data, a decision is made to permit or discontinue medicines. WHO, FDA and Roszdravnadzor regularly inform about the changes and requirements in the field of pharmacovigilance and drug turnover. Effective information exchange between medical centers, pharmaceutical companies and regulatory authorities significantly improves the quality of medical care. Pharmacovigilance training programs in clinical practice significantly increase the doctor's knowledge of the possible consequences of using drugs.

According to clinical response most commonly was developed allergy reactions $-18,2 \%$ of cases, symptoms of gastro-intestinal disorders $-14,8 \%$, in $12.5 \%$ of cases there was a cough, in $8,4 \%$-leg oedema, bleeding in $6,4 \%$ of cases. Life-threatening symptoms advanced in 5, 7\% of cases and in 3,7\% there was a wheezing (Figure 2) [3].

\section{References}

1. Dave V (2013) Current trends in Pharmacovigilance. J Pharmacovil 1: 1

2. Kantemirova BI, Timofeeva NV, Griganov VI, Shilova AA (2011) he problem of undesirable side reactions of drugs. Astrakhan Med J 6: 8-12.

3. Lukina YV, Dmitrieva NA, Zakharova AV, Zagrebelny AV, Kutishenko NP, et al. (2016) Undesirable effects of drug therapy (first results of the study according to the outpatient register profile). Ratio pharmacoth cardio 12: 306-313.

4. Wester K, Jönsson AK, Spigset O, Druid H, Hägg S (2008) Incidence of fatal adverse drug reactions: a population based study. Br J Clin Pharmacol 65: 573-579.

5. Chan A, Haw YL, Chi HH, Shun JL (2008) Cost evaluation of adverse drug reactions in hospitalized patient in Taiwan: A prospective, descriptive, observational study. Curr therap resea 69: 118-129.

6. Sultana J, Cutroneo P (2013) Clinical and economic burden of adverse drug reactions. J Pharmaco pharmacothe 4: 73-77.

7. Pirmohamed M, James S, Meakin S, Green C, Scott AK, et al.(2004) Adverse drug reactions as cause of admission to hospital: prospective analysis of 18820 patients. he BMJ 329: 15-19.

8. Lazarou J, Pomeranz BH, Corey PN (1998) Incidence of adverse drug reaction in hospitalized patients: meta-analysis of prospective studies. JAMA 279: 1200-1205.

9. Makary MA, Daniel M (2016) Medical error -the third leading cause of death in the US. BMJ 353: i2139.

10. McBride W (1977) Teratogen halidomide embryopathy. Teratolo 16

11. Lenz W (1962) halidomide and congenital abnormalities. J Lancet 279: 45-46

12. Zagorodnikova KA (2015) Organization of monitoring of the safety of medicines in the world - methodological approaches. Pacific Medi J 1: $12-15$.

13. http://www.who.int/medicines/areas/quality_safety/safety_efficacy/ advdrugreactions/en/

14. Viktorov AP (2002) Control over the safety of drugs in the world and the development of pharmacovigilance in Ukraine. he pharma 1:9-13.

15. Lepakhin VK (1998) he current state of the problem of drug safety. he international cooperation. Control of drug safety: Workshop materials: Worldwide National Congress " he Man and the Medicine", Moscow 2-15.

16. Normative legal acts in the sphere of medicinal products circulation within the framework of the Eurasian Economic Union. Pharmacovigilance. Moscow, Russia.

17. Edwards IR, Biriell C (1994) Harmonisation in pharmacovigilance. Drug Saf 10: 93-102.

18. Meyboom RH, Lindquist M, Egberts A (2000) An ABC of drug-related problems. Drug saf 22: 415-423.

19. Fattinger K, Malgorzata R, Patrice V, Clemens H, Brigitt K (2000) Epidemiology of drug exposure and adverse drug reactions in two Swiss departments of internal medicine. Br J Clin Pharmacol 49: 158-167.

20. Bouvy JC, Bruin ML, Koopmanschap MA (2015) Epidemiology of adverse drug reactions in Europe: a review of recent observational studies. Drug saf 38:437-453. 